\title{
Dense embeddings of surface groups
}

\author{
EMMANUEL BREUILLARD \\ TSACHIK GELANDER \\ JUAN SOUTO \\ PETER STORM
}

\begin{abstract}
We discuss dense embeddings of surface groups and fully residually free groups in topological groups. We show that a compact topological group contains a nonabelian dense free group of finite rank if and only if it contains a dense surface group. Also, we obtain a characterization of those Lie groups which admit a dense faithfully embedded surface group. Similarly, we show that any connected semisimple Lie group contains a dense copy of any fully residually free group.
\end{abstract}

22E40; $20 \mathrm{H} 10$

\section{Introduction}

Given a locally compact topological group $G$ and an abstract group $\Gamma$, it is natural to ask whether $\Gamma$ can be embedded densely in $G$. More generally, for a given $G$ one would like to understand its dense subgroups, and for a given $\Gamma$ one would like to know its possible completions $G$ which are topological groups. These questions are more accessible in the case where $G$ is a finite dimensional analytic Lie group over a local field. While discrete subgroups of Lie groups have been thoroughly studied for the last fifty years, very little is known about nondiscrete, and in particular, dense ${ }^{1}$ subgroups of Lie groups. A dense embedding of $\Gamma$ in $G$ may yield interesting data on $\Gamma, G$, and the spaces on which they act (Margulis [11], Sullivan [14], Gelander and Żuk [8], Lubotzky and Weiss [10], Breuillard and Gelander [4; 3], Abert and Glasner [1]).

By a surface group, we mean the fundamental group of a closed oriented surface of genus at least 2. By a free group, we mean a nonabelian free group on at least two generators. We obtain various results, all of which are proved by continuously deforming a given representation to a faithful one.

\footnotetext{
${ }^{1}$ Note that when $G$ is a connected simple Lie group, a generic subgroup with sufficiently many generators is either discrete or dense
} 
Our first result states that free groups and surface groups have the same compactifications within the category of topological groups.

Theorem 1.1 Let $G$ be a compact group. Then the following two assertions are equivalent:

- $G$ contains a dense free subgroup of finite rank.

- $G$ contains a dense surface group.

As a corollary we obtain that a compact group contains a surface group if and only if it contains a free group. It is sometimes fairly easy to verify that a given compact group contains a free subgroup by means of probabilistic methods. However, we do not know a simple characterization of the compact groups containing a dense free subgroup of finite rank. It was shown in [3] that the profinite completion $\widehat{\Gamma}$ of a finitely generated linear group $\Gamma$ contains a dense free subgroup of finite rank if and only if it is not virtually solvable (ie, contains no solvable subgroup of finite index). However, there are examples of topologically finitely generated profinite groups that satisfy a nontrivial group law (hence admit no free subgroups) although they are not virtually solvable (de Cornulier and Mann [5]). On the other hand, it is possible to verify that any connected second countable nonabelian compact group contains a dense free subgroup of rank 2 (see Proposition 8.2). Hence any such group contains also a dense surface group.

The method used to prove our Theorem 1.1 above can be pushed a little further to get a result that holds for an arbitrary locally compact group:

Theorem 1.2 Let $G$ be a locally compact group. Suppose that $G$ contains a nondiscrete free subgroup $F$ of finite rank $r>1$. Then $G$ has a subgroup $\Gamma$ containing $F$ such that $\Gamma$ is isomorphic to a surface group (of genus $2 r$ ). In particular, if $G$ has a dense free subgroup of finite rank, then it has a dense surface group.

Remark As a corollary of Theorem 1.2 we obtain an elementary proof of a result from Gelander and Glasner [7], that surface groups are primitive, ie, admit faithful primitive permutation representations. Indeed, let $\Gamma$ be a surface group and embed $\Gamma$ densely in $\operatorname{PSL}_{2}\left(\mathbb{Q}_{p}\right)$. Then $\Delta=\Gamma \cap \mathrm{PSL}_{2}\left(\mathbb{Z}_{p}\right)$ is a maximal subgroup of $\Gamma$ which contains no nontrivial normal subgroup of $\Gamma$, and the action of $\Gamma$ on $\Gamma / \Delta$ is primitive and faithful.

When $G$ is a (nondiscrete) real Lie group with a countable number of connected components, then $G$ contains a finitely generated dense free group if and only if the connected component of the identity $G^{\circ}$ is not solvable and $G / G^{\circ}$ is finitely generated $[4 ; 3]$.

We thus obtain: 
Corollary 1.3 Let $G$ be a nondiscrete real Lie group. Then the following are equivalent:

- $G$ contains a finitely generated dense free subgroup.

- $G$ contains a dense surface group.

- $G^{\circ}$ is not solvable and $G / G^{\circ}$ is finitely generated.

One key property of surface groups which motivated this research is the fact that they are fully residually free. A finitely generated group $\Gamma$ is fully residually free if for every finite set $K \subset \Gamma \backslash\{1\}$ there is a homomorphism $\phi: \Gamma \rightarrow F$ onto a free group $F$ with $K \cap \operatorname{Ker}(\phi)=\varnothing$. In other words, $\Gamma$ is fully residually free if any finite set can be separated through a surjective map onto a free group.

The class of fully residually free groups also appears in the work of Sela [13], where he shows that it coincides with his notion of limit groups. The fact that surface groups are fully residually free is due to Baumslag [2]. A group $\Gamma$ is called $d$-fully residually free if any finite set can be separated trough a surjection on $F_{d}$. Note that if $\Gamma$ is $d$-fully residually free then it is also $k$-fully residually free for any $2 \leq k<d$ (see Lemma 2.1 below).

For general fully residually free groups we prove the following:

Theorem 1.4 Let $G$ be a connected nonsolvable Lie group. Then there is a number $d=d(G)<\operatorname{dim}(G)$ (defined in Section 6) such that: if $\Gamma$ is a finitely generated $d$-fully residually free group, then there is a dense embedding $\Gamma \hookrightarrow G$.

When $G$ is topologically perfect, ie, does not surject onto the circle, then we can take $d$ to be the minimal number of generators for the Lie algebra of $G$. Since any semisimple Lie algebra is generated by 2 elements, we obtain:

Theorem 1.5 Any connected semisimple Lie group contains a dense copy of any finitely generated nonabelian fully residually free group.

Remark A group $\Gamma$ is residually free if for every $\gamma \in \Gamma \backslash\{1\}$ there is $\phi: \Gamma \rightarrow F_{d}$ with $\gamma \notin \operatorname{Ker}(\phi)$. For example, if $\Gamma$ is a surface group then $\Gamma \times \Gamma$ is residually free. Since $\mathrm{PSL}_{2}(\mathbb{C})$ does not have subgroups isomorphic to $\Gamma \times \Gamma$ we observe that in Theorem 1.4 the condition " $\Gamma$ is fully residually free" cannot be weakened to " $\Gamma$ is residually free".

Let us end this introduction by remarking that all the results obtained in this paper are concerned with the existence of subgroups with certain desired properties. However we do not obtain concrete examples. In general, this problem seems much more difficult. 
Acknowledgments E Breuillard acknowleges support from the Centre National de la Recherche Scientifique and from the Institute for Advanced Study. T Gelander has received support from NSF grant DMS-0404557 and BSF grant 2004010. P Storm has received support from an NSF Postdoctoral Research Fellowship.

\section{Eventually faithful homomorphisms and a lemma of Baum- slag}

Let $\left(\rho_{n}\right)_{n \geq 0}$ be a sequence of homomorphisms from a group $H$ to a group $G$. We say that $\left(\rho_{n}\right)_{n \geq 0}$ is eventually faithful if for every $h \in H \backslash\{1\}$ there exists an integer $n_{0}=n_{0}(h)$ such that $\rho_{n}(h) \neq 1$ for all $n \geq n_{0}$.

Since any finitely generated free group can be embedded into $F_{2}$, the free group on two generators, it follows that a finitely generated group is fully residually free if and only if it admits an eventually faithful sequence of homomorphisms to $F_{2}$.

Let us recall the following lemma of Baumslag [2].

Lemma 2.1 (Baumslag) Let $u, a_{1}, \ldots, a_{k}$ be elements of a free group $F$. Assume that $u$ does not commute with any of the $a_{i}$ 's. Then there exists $n_{0} \geq 0$ such that for all integers $n_{1}, \ldots, n_{k}$ with $\left|n_{i}\right| \geq n_{0}$ we have

$$
u^{n_{1}} a_{1} u^{n_{2}} a_{2} \cdot \ldots \cdot u^{n_{k}} a_{k} \neq 1
$$

This lemma has a few corollaries. The first one proves that surface groups are fully residually free.

Corollary 2.2 Let $\Gamma=\Gamma_{2 r}$ be the fundamental group of an orientable surface of genus $2 r(r \geq 1)$. Let us write a presentation of $\Gamma$ as

$$
\Gamma=\left\langle a_{i}, a_{i}^{\prime}, b_{i}, b_{i}^{\prime}, 1 \leq i \leq r \mid\left[a_{1}, a_{1}^{\prime}\right] \cdot \ldots \cdot\left[a_{r}, a_{r}^{\prime}\right] \cdot\left[b_{r}^{\prime}, b_{r}\right] \cdot \ldots \cdot\left[b_{1}^{\prime}, b_{1}\right]=1\right\rangle
$$

Now consider the automorphism $\sigma$ of $\Gamma$ that leaves the $a_{i}$ 's and $a_{i}^{\prime}$ 's fixed while sending every $b_{i}$ to $\gamma b_{i} \gamma^{-1}$ and every $b_{i}^{\prime}$ to $\gamma b_{i}^{\prime} \gamma^{-1}$, where $\gamma=\left[a_{1}, a_{1}^{\prime}\right] \cdot \ldots \cdot\left[a_{r}, a_{r}^{\prime}\right]$. Finally let $f$ be the surjective homomorphism from $\Gamma$ to the free group $F_{2 r}$ with free generators $x_{1}, \ldots, x_{r}$ and $x_{1}^{\prime}, \ldots, x_{r}^{\prime}$ defined by $f\left(a_{i}\right)=f\left(b_{i}\right)=x_{i}, f\left(a_{i}^{\prime}\right)=f\left(b_{i}^{\prime}\right)=$ $x_{i}^{\prime}$.

Then the sequence of maps $\left(f \circ \sigma^{n}\right)_{n \geq 0}$ is eventually faithful. 
The maps $\sigma$ and $f$ have the following simple topological interpretation. In the above classical representation of $\Gamma$ as the fundamental group of a surface, the relation gives the gluing instructions for forming a genus $2 r$ surface from a $4 r$-gon in the plane. This is demonstrated in Figure 1 for the case $r=2$. The element $\gamma$ correponds to the closed curve separating the surface into two equal parts. The map $\sigma$ corresponds to a Dehn twist around $\gamma$. The map $f$ is obtained by reflecting the surface across the separating curve $\gamma$. The image of this reflection is a surface of genus $r$ with one boundary component, whose fundamental group is freely generated by $x_{1}, \ldots, x_{r}, x_{1}^{\prime}, \ldots, x_{r}^{\prime}$.

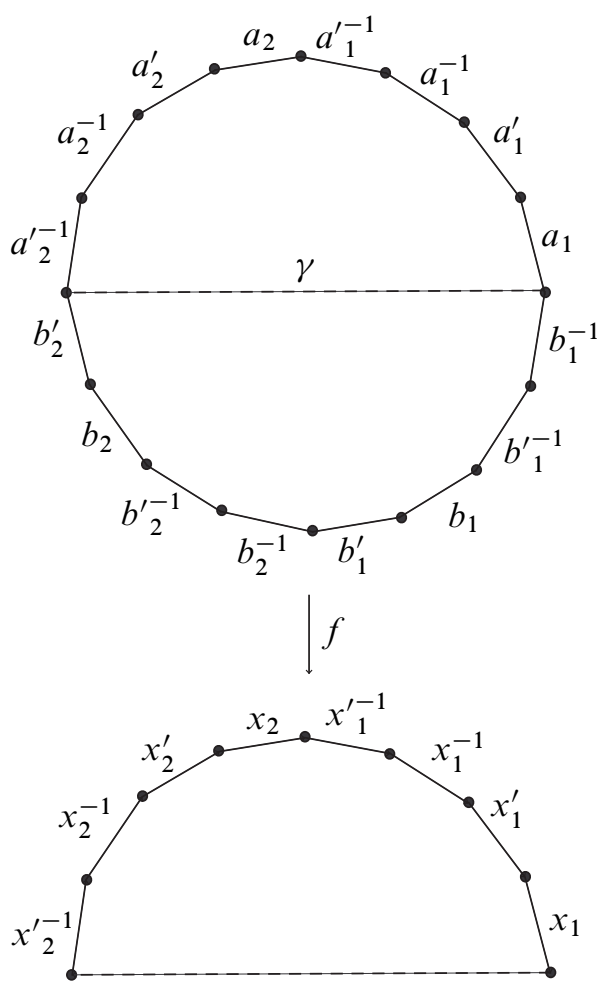

Figure 1: All curves are oriented counterclockwise. Fold the genus 4 surface across $\gamma$ to obtain $f$.

Proof Let $g \in \Gamma \backslash\{1\}$. The element $g$ can be written in the form $g=w_{1}\left(a_{i}, a_{i}^{\prime}\right)$. $w_{2}\left(b_{i}, b_{i}^{\prime}\right) \cdot . . \cdot w_{2 p-1}\left(a_{i}, a_{i}^{\prime}\right) \cdot w_{2 p}\left(b_{i}, b_{i}^{\prime}\right)$ where each $w_{i}$ is a reduced word in $2 r$ letters and the first and the last $w_{i}$ may be trivial. Up to modifying the odd $w_{2 j-1}$, we may assume that each even $w_{2 j}(1 \leq j \leq p)$ is such that $w_{2 j}\left(b_{i}, b_{i}^{\prime}\right)$ is not a power of $\gamma$. Note that the centralizer of $\gamma$ in $\Gamma$ is the cyclic group generated by $\gamma$. By regrouping several $w_{j}$ 's into a longer word if necessary, unless $g$ itself is a power of 
$\gamma$, we may also assume that $w_{2 j-1}\left(a_{i}, a_{i}^{\prime}\right)$ is not a power of $\gamma$. Let $\bar{\gamma}$ be the image of $\gamma$ under $f$. We have $f \circ \sigma^{n}(g)=w_{1} \cdot \bar{\gamma}^{n} w_{2} \bar{\gamma}^{-n} \cdot . . \cdot w_{2 p-1} \cdot \bar{\gamma}^{n} w_{2 p} \bar{\gamma}^{-n}$ where each $w_{j}=w_{j}\left(x_{i}, x_{i}^{\prime}\right)$. Since $\gamma$ does not commute with any of the $w_{j}$, Lemma 2.1 implies that $f \circ \gamma^{n}$ is eventually faithful.

The next two corollaries are very simple applications of Lemma 2.1, and are only recorded here for further use.

Corollary 2.3 Let $F$ be a free group of rank $n+1$ with free generators $x_{1}, \ldots, x_{n+1}$. Let $F^{-}$be the subgroup generated by $x_{1}, \ldots, x_{n}$. Suppose $a$ and $b$ are noncommuting elements in $F^{-}$. Consider the automorphism $\sigma$ of $F$ defined by $\sigma\left(x_{i}\right)=x_{i}$ if $i \leq n$ and $\sigma\left(x_{n+1}\right)=b x_{n+1} b^{-1}$. Let $f$ be the homomorphism of $F$ into $F^{-}$that sends each $x_{i}$ to itself for $1 \leq i \leq n$, and $x_{n+1}$ to $a$. Then the sequence of homomorphisms $\left(f \circ \sigma^{n}\right)_{n \geq 0}$ is eventually faithful.

Remark It follows from the definition that Lemma 2.1 remains true when the free group $F$ is replaced by any nonabelian fully residually free group, and in particular by a surface group.

Corollary 2.4 Let $\Gamma_{r}=\left\langle a_{i}, a_{i}^{\prime}, 1 \leq i \leq r \mid\left[a_{1}, a_{1}^{\prime}\right] \cdot \ldots \cdot\left[a_{r}, a_{r}^{\prime}\right]=1\right\rangle$ be a presentation of a surface group of genus $r$. Let $F$ be a free group of rank $2 r$ generated by $x_{i}, x_{i}^{\prime}$ for $1 \leq i \leq r$. For each integer $n \geq 0$ consider the homomorphism $\rho_{n}: F \rightarrow \Gamma_{r}$ given by $\rho_{n}\left(x_{i}\right)=a_{i}$ for $i \geq 2, \rho_{n}\left(x_{i}^{\prime}\right)=a_{i}^{\prime}$ for $i \geq 1$ and $\rho_{n}\left(x_{1}\right)=a_{1} \cdot\left(a_{1}^{\prime}\right)^{n}$. Then the sequence $\left(\rho_{n}\right)_{n \geq 1}$ is eventually faithful.

\section{Proof of Theorem 1.1}

Here we give a proof of Theorem 1.1. Let $G$ be a compact group containing a dense free group $F$ on $r$ generators. We are going to show that $G$ contains a surface group containing $F$. In order to do so, we first make sure that $G$ contains a dense free group on an even number of generators. This is done, if $r$ is odd, by enlarging $F$ in the following way. Let $a_{1}, \ldots, a_{r}$ be generators of $F$ and fix $a$ and $b$ in $F$ two noncommuting elements. Then let $B$ be the closure in $G$ of the cyclic group generated by $b$. Let $F^{+}$be the abstract free group on $r+1$ generators $y_{1}, \ldots, y_{r+1}$. To every $\beta \in B$, we associate the homomorphism $\rho_{\beta}: F^{+} \rightarrow G$ which sends each $y_{i}$ to $a_{i}$ when $1 \leq i \leq r$, and sends $y_{r+1}$ to $\beta a \beta^{-1}$. From Corollary 2.3, we know that the sequence of homomorphisms $\left(\rho_{b^{n}}\right)_{n \geq 1}$ is eventually faithful. Let $w \in F^{+} \backslash\{1\}$ and consider the set $O_{w}=\left\{\beta \in B: \rho_{\beta}(w) \neq 1\right\}$. Clearly $O_{w}$ is open in $B$. It is also dense because the set $\left\{b^{n}: n \geq n_{0}\right\}$ is dense in $B$ for any $n_{0} \geq 0$. Baire's theorem 
implies that $O=\cap_{w \in F^{+} \backslash\{1\}} O_{w}$ is dense in $B$, and is in particular nonempty. Let $\beta_{0} \in O$. The homomorphism $\rho_{\beta_{0}}$ is faithful and $\rho_{\beta_{0}}\left(F^{+}\right)$is a dense free subgroup of $G$ of rank $r+1$.

We may therefore assume that $r=2 k$. Let $x_{1}, x_{1}^{\prime}, \ldots, x_{k}, x_{k}^{\prime}$ be the $2 k$ free generators of $F$. Set $\gamma=\left[x_{1}, x_{1}^{\prime}\right] \cdot \ldots \cdot\left[x_{k}, x_{k}^{\prime}\right]$. Let $K$ be the closure in $G$ of the cyclic group generated by $\gamma$. Keeping the same notation as in Corollary 2.2 for the presentation of the surface group $\Gamma_{2 r}$, we define for every $\alpha \in K$ a homomorphism $\sigma_{\alpha}: \Gamma_{2 r} \rightarrow G$ by sending $a_{i}$ to $x_{i}, a_{i}^{\prime}$ to $x_{i}^{\prime}, b_{i}$ to $\alpha x_{i} \alpha^{-1}$ and $b_{i}^{\prime}$ to $\alpha x_{i}^{\prime} \alpha^{-1}$. From Corollary 2.2, we know that the sequence of homomorphisms $\left(\sigma_{\gamma^{n}}\right)_{n \geq 1}$ is eventually faithful. As above, let $w \in \Gamma_{2 r} \backslash\{1\}$ and consider the set $U_{w}=\left\{\alpha \in K \mid \sigma_{\alpha}(w) \neq 1\right\}$. Clearly $U_{w}$ is open in $K$. It is also dense, because for any $n_{0} \geq 0$ the set $\left\{\gamma^{n}: n \geq n_{0}\right\}$ is dense in $K$. Applying Baire's theorem, we obtain that $U:=\cap_{w \in F^{+} \backslash\{1\}} U_{w}$ is dense in $K$ and in particular nonempty. Let $\alpha_{0} \in U$. Note that for every $\alpha \in K$ the image $\sigma_{\alpha}\left(\Gamma_{2 r}\right)$ is dense in $G$ because it contains $F$ as a subgroup. The homomorphism $\sigma_{\alpha_{0}}$ is faithful and $\sigma_{\alpha_{0}}\left(\Gamma_{2 r}\right)$ is a surface group densely embedded in $G$.

We now pass to the converse statement. Let $\Gamma_{r}$ be a dense surface group of genus $r$ in $G$. Keep the notation of Corollary 2.4 and let $A$ be the closure in $G$ of the cyclic group generated by $a_{1}^{\prime}$. For every $\omega \in A$ let $\pi_{\omega}: F_{2 r} \rightarrow G$ be the homomorphism that sends $x_{1}$ to $a_{1} \omega$, while for $i \geq 2, x_{i}$ is sent to $a_{i}$ and for $i \geq 1, x_{i}^{\prime}$ is sent to $a_{i}^{\prime}$. According

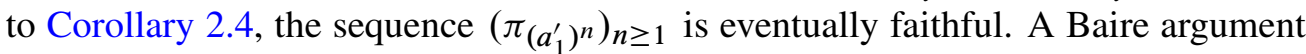
similar to the one above show that $\pi_{\omega_{0}}$ is faithful for some $\omega_{0} \in A$. It remains to check that $\pi_{\omega_{0}}\left(F_{2 r}\right)$ is dense in $G$. This is clear because it contains all $a_{i}$ 's for $i \geq 2$ and $a_{i}^{\prime}$ 's for $i \geq 1$. In particular $\pi_{\omega_{0}}\left(F_{2 r}\right)$ contains $a_{1}^{\prime}$, hence the closure of $\pi_{\omega_{0}}\left(F_{2 r}\right)$ must contain $\omega_{0}$. Therefore the closure of $\pi_{\omega_{0}}\left(F_{2 r}\right)$ must contain $a_{1}$, implying it is all of $G$. This completes the proof of Theorem 1.1.

\section{Proof of Theorem 1.2}

Let us make the obvious remark that there are (non-locally compact) Hausdorff nondiscrete topological groups where the property of Theorem 1.2 does not hold. For instance consider the free group with the induced topology coming from a dense embedding inside a compact Lie group.

Let $x_{1}, \ldots, x_{r}$ be the $r$ free generators of the nondiscrete free subgroup $F$. As in the compact case, we are first going to enlarge the free subgroup $F$ (of rank $r$ ) to a bigger free subgroup of rank $2 r$ by adding $r$ free generators, then deform that free subgroup into a surface group. 
By the structure theory of locally compact groups (Van Dantzig's theorem, see Montgomery and Zippin [12]) $G$ has an open subgroup $H^{\prime}$ containing the connected component of the identity $G^{\circ}$ in such a way that $H^{\prime} / G^{\circ}$ is compact. Moreover there is a normal compact subgroup $K$ of $H^{\prime}$ such that $H^{\prime} / K$ is a Lie group, and $K$ can be chosen sufficiently small for the finite set of conjugates $\left\{K_{i}=x_{i} K x_{i}^{-1}\right\}_{i=1}^{r}$ to be contained in the open finite intersection $\cap_{i=1}^{r} x_{i} H^{\prime} x_{i}^{-1}$ [12, Theorem 4.6]. This second assumption is used only to know that the set $K K_{1} \ldots K_{r}$ is a subgroup of $G$. Up to replacing $H^{\prime}$ by a smaller open subgroup $H \leq G$ we can assume that $H / K$ is connected.

Let $U$ and $V$ be sufficiently small neighborhoods of the identity in $H$ so that $x_{i} u x_{i}^{-1} u^{-1} \in V$ for any $u \in U$ and $i=1, \ldots, r$, and so that the projection of any element in $V^{r}$ lies in a 1-parameter subgroup of $H / K$. We are going to find elements $x_{1}^{\prime}, \ldots, x_{r}^{\prime}$ in $U$ which, together with the $x_{i}$ 's, form $2 r$ free generators of a free subgroup of $G$.

For this purpose, pick two noncommuting elements $a$ and $b$ in $F$ that are in $U$ modulo $K$. This is always possible because $F$ is not discrete. The proof will have two cases. Case (I) is when $F \cap K \neq\{1\}$. Case (II) is when $F \cap K=\{1\}$. In case (I) we can clearly assume that $a$ and $b$ belong to $K$. (Pick an element in $F \cap K$ and some suitable conjugate of it.) Suppose that $x_{1}^{\prime}, \ldots, x_{j}^{\prime}$ have been constructed. Define $F_{r+j+1}$ to be an abstract free group on $r+j+1$ generators $y_{1}, \ldots, y_{r}, y_{1}^{\prime}, \ldots, y_{j+1}^{\prime}$, and let us find $x_{j+1}^{\prime}$. We will handle the two cases separately.

Assume first we are in case (I). Let $B \leq K$ be the closure of the cyclic group generated by $b$. For $\beta \in B$ let $\rho_{\beta}$ be the homomorphism sending each $y_{i}$ to $x_{i}$, each $y_{i}^{\prime}$ to $x_{i}^{\prime}$ for $i \leq j$, and $y_{j+1}^{\prime}$ to $\beta a \beta^{-1}$. Corollary 2.3 and Baire's theorem ensure that the subset of those $\beta$ for which $\rho_{\beta}$ is faithful is Baire dense in $B$, and hence nonempty. Fix such an element $\beta_{0} \in B$. The desired new generator is $x_{j+1}^{\prime}=\rho_{\beta_{0}}\left(y_{j+1}^{\prime}\right)$.

Now assume we are in case (II). Morally, we repeat the argument of case (I), but the details differ. By induction we may assume the free group $\left\langle x_{1}, \ldots, x_{r}, x_{1}^{\prime}, \ldots, x_{j}^{\prime}\right\rangle$ intersects $K$ trivially. Let $B$ be the 1-parameter subgroup of $H / K$ containing the coset $b K$. For $\beta \in B$ let $\rho_{\beta}$ be the map to $H / K$ sending each $y_{i}$ to $x_{i} K$, each $y_{i}^{\prime}$ to $x_{i}^{\prime} K$ for $i \leq j$, and $y_{j+1}^{\prime}$ to $\beta \bar{a} \beta^{-1}$, where $\bar{a}=a K$. This yields a one parameter family of representations from $\left\langle y_{1}, \ldots, y_{r}, y_{1}^{\prime}, \ldots, y_{j+1}^{\prime}\right\rangle$ to $H / K$. For any word $w$ in $\left\langle y_{1}, \ldots, y_{r}, y_{1}^{\prime}, \ldots, y_{j+1}^{\prime}\right\rangle$, the set $\left\{\beta \in B: \rho_{\beta}(w) \neq 1\right\}$ is open because the map $\mathrm{ev}_{w}: \beta \mapsto \rho_{\beta}(w)$ is continuous. By Corollary 2.3 this continuous map ev $w$ is not constant. Now we use the fact that $B$ and $H / K$ are Lie groups, which implies they are real analytic manifolds and $\mathrm{ev}_{w}$ is real analytic. Therefore the closed $\operatorname{set}^{\mathrm{ev}} \mathrm{v}_{w}^{-1}(1)$ is nowhere dense. By Baire's theorem the subset of $\beta \in B$ for which $\rho_{\beta}$ is faithful is 
nowhere dense in $B$. Fix such a $\beta_{0} \in B$ sufficiently near the identity for there to be an element $x_{j+1}^{\prime}$ contained in the intersection $\rho_{\beta_{0}}^{-1}\left(y_{j+1}^{\prime}\right) \cap U \subset G$. This choice of $x_{j+1}^{\prime}$ completes the induction in case (II).

Continuing the argument in both cases, call $F^{\prime}$ the new free subgroup on $2 r$ generators. Note that $F \leq F^{\prime}$, and in case (II) we have $F^{\prime} \cap K=\{1\}$. Consider the product of commutators $\gamma=\left[x_{1}, x_{1}^{\prime}\right] \cdot \ldots \cdot\left[x_{r}, x_{r}^{\prime}\right]$. Let $\Gamma_{2 r}$ be a surface group given with the presentation written above in (1). Consider the centralizer $Z_{G}(\gamma)$ of $\gamma$ in $G$. Given an element $\alpha$ in $Z_{G}(\gamma)$ we can define a representation $\rho_{\alpha}: \Gamma_{2 r} \rightarrow G$ by setting $\rho_{\alpha}\left(a_{i}\right)=x_{i}, \rho_{\alpha}\left(a_{i}^{\prime}\right)=x_{i}^{\prime}, \rho_{\alpha}\left(b_{i}\right)=\alpha x_{i} \alpha^{-1}$, and $\rho_{\alpha}\left(b_{i}^{\prime}\right)=\alpha x_{i}^{\prime} \alpha^{-1}$. Corollary 2.2 shows that the sequence $\left(\rho_{\gamma^{n}}\right)_{n \geq 1}$ is eventually faithful. We will make use of the following lemmas:

Lemma 4.1 Let $H$ be a locally compact group and $K$ a compact normal subgroup such that $H / K$ is a Lie group. Let $\{x(t)\}_{t}$ be a 1 -parameter subgroup in $H / K$. Then $\{x(t)\}_{t}$ can be lifted to a 1 -parameter subgroup $\{\widetilde{x}(t)\}_{t}$ in $H$ such that $\pi(\widetilde{x}(t))=x(t)$ where $\pi: H \rightarrow H / K$ is the quotient map.

Proof See the end of Section 4.7 of [12].

Lemma 4.2 Let $H$ be a locally compact group and $K$ a compact normal subgroup such that $H / K$ is connected. Then $H=Z_{H}(K) K$ where $Z_{H}(K)$ is the centralizer of $K$ in $H$.

Proof It follows from Lemma 4.1 that $H=H^{\circ} K$.

Let $\rho: H \rightarrow \operatorname{Aut}(K)$ be a map that sends $h \in H$ to the automorphism i $(h)$ of $K$ given by the conjugation by $h$. Then $\operatorname{ker} \rho=Z_{H}(K)$. We need to show that $\rho(H)=\rho(K)$ and for this it is clearly enough to prove that $\rho\left(H^{\circ}\right)$ is contained in $\operatorname{Inn}(K)$, the group of inner automorphisms of $K$. This is a consequence of the following lemma:

Lemma 4.3 Let $K$ be a compact group. Then the connected component of the identity of $\operatorname{Aut}(K)$ is contained in $\operatorname{Inn}(K)$.

Proof First assume that $K$ is a Lie group. Then $K \simeq D \times T \times S$ where $S$ is semisimple, $T$ a torus and $D$ is a finite group. As is well-known, $\operatorname{Inn}(S)$ has finite index in $\operatorname{Aut}(S)$, and $\operatorname{Aut}(T)$ is discrete. It follows easily that $\operatorname{Aut}(K)^{\circ} \leq \operatorname{Inn}(S)$. Now we pass to the general case.

According the Peter-Weyl theorem, $K$ has a descending chain of compact normal subgroups $C_{1} \supset C_{2} \supset C_{3} \supset \ldots$ such that any open neighborhood of the identity 
contains all but a finite number of the subgroups $\left\{C_{i}\right\}$, and the quotient $K / C_{i}$ is always a Lie group. By pulling back a small identity neighborhood from $K / C_{i}$ to $K$ we obtain an open set $U_{i} \subset K$ containing $C_{i}$ such that any subgroup of $K$ inside $U_{i}$ is in fact contained in $C_{i}$. Therefore by connectivity every automorphism in $\operatorname{Aut}(K)^{\circ}$ preserves $C_{i}$. This yields a map

$$
\operatorname{Aut}(K)^{\circ} \rightarrow \operatorname{Aut}\left(K / C_{i}\right)^{\circ} \leq \operatorname{Inn}\left(K / C_{i}\right) .
$$

Let $\phi \in \operatorname{Aut}(K)^{\circ}$. For each $i$ pick an element $h_{i} \in K$ such that $\phi(g) C_{i}=h_{i} g h_{i}^{-1} C_{i}$ for all $g \in K$. Since the subgroups $\left\{C_{i}\right\}$ become arbitrarily small it follows that $\mathrm{i}\left(h_{i}\right) \rightarrow \phi$ in the compact-open topology on $\operatorname{Aut}(K)$. Since $\operatorname{Inn}(K)$ is a closed subgroup of $\operatorname{Aut}(K)$ it follows that $\phi$ is an inner automorphism. This completes the proof of Lemmas 4.2 and 4.3.

Let us return to the proof of Theorem 1.2. Suppose we are in case (I). Then $\gamma$ is contained in the compact subgroup $K K_{1} \ldots K_{r} \leq G$, where $K_{i}=x_{i} K x_{i}^{-1}$. Let $A$ be the closure in $G$ of the cyclic group generated by $\gamma$. By our assumption, $A$ is compact. Then $A \leq Z_{G}(\gamma)$, and by Corollary 2.2, if $w$ is a nontrivial element in $\Gamma_{2 r}$ then $\left\{\alpha \in A: \rho_{\alpha}(w) \neq 1\right\}$ is an open dense subset of $A$. By Baire's theorem there is an $\alpha_{0} \in A$ such that $\rho_{\alpha_{0}}$ is faithful. Its image contains $F$ and is isomorphic to the surface group $\Gamma_{2 r}$. This completes the proof in case (I).

Finally suppose that we are in case (II). Then $F^{\prime} \cap K=\{1\}$ and the sequence ( $\pi \circ$ $\left.\rho_{\gamma^{n}}\right)_{n \geq 1}$ is eventually faithful, where $\pi: H \rightarrow H / K$ is the projection map. Let $\pi(\gamma)=$ $\beta(1)$ where $\{\beta(t)\}_{t}$ is a 1-parameter subgroup of $H / K$. The centralizer $Z_{H}(K)$ is closed in $H$, hence locally compact, and by Lemma $4.2, H / K \cong Z_{H}(K) / K \cap Z_{H}(K)$. Lemma 4.1 ensures that $\{\beta(t)\}_{t}$ can be lifted to a 1 -parameter subgroup $\{c(t)\}_{t}$ in $Z_{H}(K)$, ie, $\beta(t)=c(t) K$. We now claim that $\{c(t)\}_{t} \leq Z_{G}(\gamma)$.

Indeed, by Lemma 4.2, we can write $\gamma=c k$ where $c \in Z_{H}(K)$ and $k \in K$. However $\pi(\gamma)=\beta(1)$, hence $c(1) K=\gamma K$, hence $c(1) k^{\prime}=c$ for some $k^{\prime} \in K$. But for each $t$, $c(t)$ commutes with $c(1)$ and with $k^{\prime}$, hence it commutes with $c$. As $c(t) \in Z_{H}(K)$ it must also commute with $k$, hence with $\gamma$. This proves the claim.

As a consequence, we obtain a one parameter family of representations $\rho_{t}: \Gamma_{2 r} \rightarrow G$ by setting $\rho_{t}:=\rho_{c(t)}$. Again $\left\{t \in \mathbb{R}: \pi \circ \rho_{t}(w) \neq 1\right\}$ is open because $t \mapsto \rho_{t}(w)$ is continuous from $\mathbb{R}$ to $G$. By Corollary 2.2, the sequence $\pi \circ \rho_{n}=\pi \circ \rho_{\gamma^{n}}$ is eventually faithful. This implies that the analytic map $t \mapsto \pi \circ \rho_{t}(w)$ from $\mathbb{R}$ to the Lie group $H / K$ is not constant. Therefore the set $\left\{t \in \mathbb{R}: \pi \circ \rho_{t}(w) \neq 1\right\}$ is dense. Again, by Baire's theorem there must be a $t_{0} \in \mathbb{R}$ such that $\pi \circ \rho_{t_{0}}$ is faithful. Then $\rho_{t_{0}}$ is also faithful and its image is a subgroup of $G$ isomorphic to $\Gamma_{2 r}$ containing $F$. This completes the proof of Theorem 1.2. 


\section{The analytic structure of $\operatorname{Hom}(\Gamma, G)$}

In this section we will recall some facts about the structure of $\operatorname{Hom}(\Gamma, G)$ as an analytic variety, where $\Gamma$ is a finitely generated group and $G$ is a Lie group.

Consider first the case that $\Gamma$ is isomorphic to a free group $F_{k}$ with free basis $e_{1}, \ldots, e_{k}$. A homomorphism $\sigma \in \operatorname{Hom}\left(F_{k}, G\right)$ is determined by $\sigma\left(e_{1}\right), \ldots, \sigma\left(e_{k}\right)$ and hence we have an identification of $\operatorname{Hom}\left(F_{k}, G\right)$ with the analytic manifold $G^{k}=G \times \cdots \times G$. Given an element $\gamma=e_{i_{1}} \ldots e_{i_{l}} \in F_{k}$ we consider the analytic map

$$
P_{\gamma}: G^{k} \rightarrow G, \quad P_{\gamma}\left(A_{1}, \ldots, A_{k}\right)=A_{i_{1}} \cdots A_{i_{l}} .
$$

The set $P_{\gamma}^{-1}\left(1_{G}\right)=\left\{\rho \in \operatorname{Hom}\left(F_{k}, G\right): \gamma \in \operatorname{Ker}(\rho)\right\}$ is a closed analytic subvariety of $\operatorname{Hom}\left(F_{k}, G\right)$. Recall the following basic result (Epstein [6]):

Theorem 5.1 Let $G$ be a connected nonsolvable Lie group. Then the set of faithful homomorphisms $\sigma: F_{k} \rightarrow G$ is dense and has full Haar measure in $\operatorname{Hom}\left(F_{k}, G\right) \cong G^{k}$.

We include a proof for the convenience of the reader.

Proof By definition $\cup_{\gamma \in F_{k} \backslash 1_{F_{k}}} P_{\gamma}^{-1}\left(1_{G}\right)$ is the complement of the set of faithful representations. The claim follows from the Baire category theorem if $P_{\gamma}^{-1}\left(1_{G}\right)$ is nowhere dense and of 0 measure for all $\gamma \neq 1_{\Gamma}$. Since $P_{\gamma}^{-1}\left(1_{G}\right)$ is an analytic subvariety of $G^{k}$, it is either nowhere dense and of 0 measure or contains $G^{k}$ since $G$ is connected. To show that the later case cannot occur, it suffices to find one faithful representation, ie, it suffices to find a nonabelian free subgroup of $G$. The existence of a free subgroup in $G$ follows from the Tits alternative [15], or more simply from the fact that $G$ contains a subgroup locally isomorphic to either $\operatorname{PSL}_{2}(\mathbb{R})$ or $\operatorname{PSO}(3)$, and each of these groups contains a free subgroup.

Now let $\Gamma$ be a general finitely generated group. To a given presentation $\Gamma=$ $\left\langle\gamma_{1}, \ldots, \gamma_{k} \mid\left\{R_{i}\right\}_{i \in I}\right\rangle$ of $\Gamma$, we associate the surjection $\pi: F_{k} \rightarrow \Gamma$ defined by $\pi\left(e_{j}\right)=\gamma_{j}$. The homomorphism $\pi$ induces an injective map

$$
\pi^{*}: \operatorname{Hom}(\Gamma, G) \rightarrow \operatorname{Hom}\left(F_{k}, G\right)
$$

and its image coincides with $\cap_{i \in I} P_{R_{i}}^{-1}\left(1_{G}\right)$. Hence, we can identify $\operatorname{Hom}(\Gamma, G)$ with an analytic subvariety of $G^{k}$. (In fact, the induced structure of $\operatorname{Hom}(\Gamma, G)$ as an analytic variety does not depend on the presentation of $\Gamma$. We will not use this.) An important observation is that for all $\gamma \in \Gamma$ the map $P_{\gamma}^{\Gamma}$ : $\operatorname{Hom}(\Gamma, G) \rightarrow G$ given by $P_{\gamma}^{\Gamma}(\rho)=\rho(\gamma)$ is analytic. Moreover, if $[\gamma] \in F_{k}$ is an element representing $\gamma$ then 
we have $P_{\gamma}^{\Gamma}=\left.P_{[\gamma]}\right|_{\operatorname{Hom}(\Gamma, G)}$. This is why in the sequel we will simplify notation and write $P_{\gamma}^{\Gamma}=P_{\gamma}$.

An important fact for our considerations is that analytic subvarieties admit locally finite stratifications with smooth strata. The following crucial result is due to Whitney, Thom and Lojasiewicz. We refer to Kaloshin [9] for its proof.

Proposition 5.2 Let $V$ be an analytic subvariety of an analytic manifold $M$. Then there is a locally finite decomposition $V=\cup V_{i}$, where $V_{i}$ are connected analytic submanifolds of $M$.

The statement of Proposition 5.2 is much weaker than, and follows directly from, [9, Theorem 1]. We have chosen this simplified statement to avoid recalling the more subtle properties of stratifications. Despite this, we will refer to the submanifolds $V_{i}$ as the strata of $V$.

\section{Dense subgroups of connected Lie groups}

This section will establish some properties of dense subgroups of connected Lie groups. We begin by recalling some results from [4]. We then determine the number $d(G)$ of Theorem 1.4, and finally study the structure of the set $\mathcal{D}\left(F_{k}, G\right)$ of dense representations of the free group $F_{k}$ in $G$.

A Lie group $H$ is topologically perfect if its commutator group is dense. Recall the following theorem from [4] (see also [8]):

Theorem 6.1 Let $H$ be a connected topologically perfect Lie group. Assume that the Lie algebra $\operatorname{Lie}(H)$ is generated (as a Lie algebra) by $d=d(H)$ elements. Then there is an identity neighborhood $U \subset H$, and a proper analytic subvariety $R \subset U^{d}$, such that $\left\langle h_{1}, \ldots, h_{d}\right\rangle$ is dense in $H$ for any $\left(h_{1}, \ldots, h_{d}\right) \in U^{d} \backslash R$.

When $G$ is topologically perfect we can define the constant $d(G)$ of Theorem 1.4 to be the minimal number of generators for $\operatorname{Lie}(G)$. As a consequence of Theorem 6.1 we obtain:

Corollary 6.2 Let $G$ be a connected topologically perfect Lie group. Then $\mathcal{D}(\Gamma, G)$ is open in $\operatorname{Hom}(\Gamma, G)$, and dense in a neighborhood of the trivial representation.

Proof If $\rho_{0} \in \operatorname{Hom}(\Gamma, G)$ is a representation of $\Gamma$ in $G$ with dense image, then for some $\gamma_{1}, \ldots, \gamma_{d} \in \Gamma$, where $d=d(G)$, we have $\left(\rho_{0}\left(\gamma_{1}\right), \ldots, \rho_{0}\left(\gamma_{d}\right)\right) \in U^{d} \backslash R$. But then $\left(\rho\left(\gamma_{1}\right), \ldots, \rho\left(\gamma_{d}\right)\right) \in U^{d} \backslash R$ for any $\rho$ sufficiently close to $\rho_{0}$ in $\operatorname{Hom}(\Gamma, G)$. By Theorem 6.1 any such $\rho$ has a dense image. 
We now define $d(G)$ for a general connected Lie group $G$.

For a connected abelian Lie group $A$, define $\operatorname{rank}(A)$ as the dimension of the tensor with $\mathbb{R}$, ie, if $\mathbb{T}$ is the one dimensional torus and $A=\mathbb{T}^{j} \times \mathbb{R}^{k}$ then $\operatorname{rank}(A)=k$. It is easy to see that a generic set (in the sense of the Baire category theorem or measure theory) of $k+1$ elements generates a dense subgroup in $A$. For example, if A is compact then a generic element generates a dense cyclic subgroup. In general, $k+1$ elements $a_{1}, \ldots, a_{k+1} \in A$ generate a dense subgroup if and only if the projections of the first $k$ elements $a_{1}, \ldots, a_{k}$ to the second factor $\mathbb{R}^{k}$ form a basis, and, after identifying the compact quotient $A /\left\langle a_{1}, \ldots, a_{k}\right\rangle$ with $\mathbb{T}^{j+k}$, the $j+k$ coordinates of the projection of the last element $a_{k+1}$ to this torus are independent. For a connected abelian Lie group $A$, we thus define $d(A)=\operatorname{rank}(A)+1$.

Let now $G$ be a general connected Lie group. Set $G_{0}=G$ and define inductively $G_{i}$ to be the closure of the derived group $\left[G_{i-1}, G_{i-1}\right]$. (In more standard notation, the subgroup $G_{i}$ is denoted by the mildly cumbersome $\overline{G^{(i)}}$, which will not be used here.) The decreasing sequence $G_{i}$ must stabilize after finitely many steps $m$ to a group $H=G_{m}$, and $H$ has the property that its commutator is dense, ie, it is topologically perfect. The general case is reduced to the abelian and topologically perfect cases using the following:

Lemma 6.3 A subgroup $D$ of $G$ is dense if and only if

(1) its image in $G / G_{2}$ is dense in $G / G_{2}$, and

(2) its intersection with $H$ is dense in $H$.

Proof If $D$ is dense then (1) follows immediately. Moreover the commutator group $[D, D]$ is clearly dense in $G_{1}$, and by a simple induction the $m^{\text {th }}$ commutator of $D$ is dense in $H=G_{m}$.

The other direction will follow if we can show that (1) implies the image of $G$ in $G / H$ is dense in $G / H$. To do this we will use the fact that $G / H$ is solvable. In a connected solvable Lie group $B$, a subgroup is dense if and only if its image in $B / B_{2}$ (modulo the second closed commutator) is dense in $B / B_{2}$. To see this, note that the commutator of a connected solvable Lie group is nilpotent, and that a subgroup of a nilpotent group is dense if and only if it is dense modulo the first commutator.

We define the number $d(G)$ as follows ${ }^{2}$ :

$$
d(G)=\max \left\{d\left(G_{0} / G_{1}\right), d\left(G_{1} / G_{2}\right), d(H)\right\},
$$

\footnotetext{
${ }^{2}$ In case $G / H$ is nilpotent, one can take $d(G)=\max \left\{d\left(G_{0} / G_{1}\right), d(H)\right\}$.
} 
where $d(H)$ is the minimal number of generators for the Lie algebra of $H$, and $d\left(G_{i} / G_{i+1}\right)=\operatorname{rank}\left(G_{i} / G_{i+1}\right)+1$.

Consider the following subsets of $\operatorname{Hom}(\Gamma, G)$ :

$$
\begin{gathered}
\mathcal{D}_{H}(\Gamma, G)=\{\rho \in \operatorname{Hom}(\Gamma, G): \overline{\rho(\Gamma) \cap H}=H\}, \text { and } \\
\mathcal{D}_{G / G_{2}}(\Gamma, G)=\left\{\rho \in \operatorname{Hom}(\Gamma, G): \overline{\rho(\Gamma) G_{2}}=G\right\} .
\end{gathered}
$$

By Lemma 6.3 we have:

$$
\mathcal{D}(\Gamma, G)=\mathcal{D}_{H}(\Gamma, G) \cap \mathcal{D}_{G / G_{2}}(\Gamma, G) .
$$

Moreover, for free groups we have:

Lemma 6.4 Suppose that $k \geq d(G)$, then:

- The set $\mathcal{D}_{H}\left(F_{k}, G\right)$ is open in $\operatorname{Hom}\left(F_{k}, G\right)$.

- The set $\mathcal{D}_{G / G_{2}}\left(F_{k}, G\right)$ is the complement of a countable union of proper closed analytic subvarieties of $\operatorname{Hom}\left(F_{k}, G\right)$. In particular, it is of second category.

Proof The first claim follows from Theorem 6.1: if $\rho_{0} \in \mathcal{D}_{H}\left(F_{k}, G\right)$, then $\rho\left(F_{k}\right)_{m}$ is dense in $G_{m}=H$. Hence there are $d(H)$ words involving commutators of length $m$ in $k$ letters, such that when applying them to the image (under $\rho_{0}$ ) of the generators of $F_{k}$ (think of them as the coordinates of a point in $G^{d(H)}$ ) one gets a point in $U^{d(H)} \backslash R \subset H^{d(H)}$. Clearly if $\rho$ is sufficiently close to $\rho_{0}$ then the same words applied to the $\rho$ image of the generators still yield a point in $U^{d(H)} \backslash R$. By Theorem 6.1, $\rho\left(F_{k}, G\right) \cap H$ is dense in $H$.

To see the second claim note that a subgroup of $G / G_{2}$ is dense in $G / G_{2}$ if its image in $G / G_{1}$ is dense in $G / G_{1}$ and its intersection with $G_{1}$ projects to a dense subgroup of $G_{1} / G_{2}$. Both conditions are generic in the sense that their complements are a countable union of proper analytic closed subvarieties: there are $d\left(G_{0} / G_{1}\right)$ words with $k$ letters which generically generate a dense subgroup in the quotient $G_{0} / G_{1}$, and $d\left(G_{1} / G_{2}\right)$ words involving commutators of the $k$ letters which generically generate a dense subgroup in the quotient $G_{1} / G_{2}$. The former assertion is clear, while the latter is a little harder to see and we leave it to the reader as an exercise. In fact, if $x_{1}, \ldots, x_{k}$ are generic elements of $\mathrm{G}$ then the commutators $\left[x_{1}, x_{i}\right]$ for $2 \leq i \leq k$ form a basis of $G_{1} / G_{2}$, and together with $\prod_{i=2}^{k}\left[x_{1}^{i}, x_{i}\right]$ they generate a dense subgroup of $G_{1} / G_{2}$.

Corollary 6.5 Assume $G$ is a connected nonsolvable Lie group. If $k \geq d(G)$ then there exists a sequence of faithful representations $\left(\sigma_{i}\right) \subset \mathcal{D}\left(F_{k}, G\right)$ converging to the trivial representation. 
Proof By Corollary 6.2 there exists a sequence $\left(\sigma_{i}^{\prime}\right) \subset \mathcal{D}\left(F_{k}, H\right)$ converging to the trivial representation. By Lemma 6.4 and Theorem 5.1, it is possible to obtain the desired sequence $\left(\sigma_{i}\right)$ via an arbitrarily small perturbation of $\left(\sigma_{i}^{\prime}\right)$.

\section{Proof of Theorem 1.4}

We are now in a position to complete the proof of Theorem 1.4. We begin by fixing once and for all a relatively compact open neighborhood $B \subset \operatorname{Hom}(\Gamma, G)$ of the trivial homomorphism and let $V_{1}, \ldots, V_{s}$ be finitely many strata of $\operatorname{Hom}(\Gamma, G)$ covering $B$.

The group $\Gamma$ is, as assumed, $d$-fully residually free for $d=d(G)$. Hence it is generated by $k \geq d$ elements and there is a sequence of surjective homomorphisms $\phi_{i}: \Gamma \rightarrow F_{d}$ such that for every $\gamma \in \Gamma \backslash 1_{\Gamma}$ there is $i_{\gamma}$ with $\gamma \notin \operatorname{Ker}\left(\phi_{i}\right)$ for all $i \geq i_{\gamma}$. The assumption that $\Gamma$ is nonabelian implies that $d \geq 2$. The homomorphisms $\phi_{i}$ induce analytic maps $\phi_{i}^{*}: \operatorname{Hom}\left(F_{d}, G\right) \rightarrow \operatorname{Hom}(\Gamma, G)$. By Corollary 6.5 we can choose a sequence $\left(\sigma_{i}\right) \subset \operatorname{Hom}\left(F_{d}, G\right)$ of faithful representations with dense image sufficiently close enough to the trivial homomorphism so that $\phi_{i}^{*}\left(\sigma_{i}\right) \in B$ for all $i$. Up to passing to a subsequence and relabelling, we may assume that $\phi_{i}^{*}\left(\sigma_{i}\right) \in V_{1} \subset \operatorname{Hom}(\Gamma, G)$ for all $i$.

Given $\gamma \in \Gamma \backslash 1_{\Gamma}$ we deduce from the connectivity of $V_{1}$, using analytic continuation and the implicit functions theorem, that either $V_{1} \subset\left\{P_{\gamma}^{-1}\left(1_{G}\right)\right\}$ or $V_{1} \cap\left\{P_{\gamma}^{-1}\left(1_{G}\right)\right\}$ is nowhere dense in $V_{1}$. The former case cannot occur since by construction we have $P_{\gamma}\left(\phi_{i}^{*}\left(\sigma_{i}\right)\right)=\sigma_{i}\left(\phi_{i}(\gamma)\right) \neq 1_{G}$ for all $i$ sufficiently large. In particular, Baire's category theorem implies that the set $\mathcal{F}(\Gamma, G) \cap V_{1}$ of all faithful $\rho \in V_{1}$ is of second category in $V_{1}$.

By construction the image of $\phi_{1}^{*}\left(\sigma_{1}\right)$ coincides with the image of $\sigma_{1}$ and hence is dense. This implies that the open subset $\mathcal{D}_{H}(\Gamma, G) \cap V_{1}$ of $V_{1}$ is nonempty. Additionally it implies that the set $\mathcal{D}_{G / G_{2}}(\Gamma, G) \cap V_{1}$ is nonempty. Since it is the complement of a countable union of proper closed analytic subvarieties we conclude, again by analyticity and the implicit functions theorem, that all these varieties are proper, and that $\mathcal{D}_{G / G_{2}}(\Gamma, G) \cap V_{1}$ is also of second category. Hence the intersection

$$
\mathcal{F}(\Gamma, G) \cap \mathcal{D}(\Gamma, G) \cap V_{1}=\mathcal{F}(\Gamma, G) \cap \mathcal{D}_{G / G_{2}}(\Gamma, G) \cap \mathcal{D}_{H}(\Gamma, G) \cap V_{1}
$$

is not empty because it is the intersection of the second category subset

$$
\mathcal{F}(\Gamma, G) \cap \mathcal{D}_{G / G_{2}}(\Gamma, G) \cap V_{1}
$$

with the nonempty open subset $\mathcal{D}_{H}(\Gamma, G) \cap V_{1}$ of $V_{1}$. 


\section{Some remarks on connected compact groups}

When $G$ is a connected compact Lie group then $d(G)=1$ if $G$ is abelian and $d(G)=2$ if it is not. For compact semisimple Lie groups one can easily deduce the following lemma from Theorem 6.1. The general case follows by an simple argument similar to the one given in the proof of Lemma 6.4.

Lemma 8.1 Let $G$ be a connected compact Lie group. Then the set $\mathcal{D}\left(F_{2}, G\right)$ is of full Haar measure and Baire dense in $\operatorname{Hom}\left(F_{2}, G\right) \cong G \times G$.

When $G$ is nonabelian, Theorem 5.1 says that a generic pair $(a, b) \in G \times G$ also generates a free group. We shall now generalize this result to an arbitrary compact connected group.

Proposition 8.2 Let $G$ be a second countable connected compact nonabelian group. Then there exists a subset $\mathcal{O}$ in $G \times G$, which is both of second Baire category and of full Haar measure, such that any pair $(a, b)$ in $\mathcal{O}$ generates a dense free subgroup in $G$.

Proof By the Peter-Weyl theorem (c.f. [12]) there is a decreasing sequence of normal compact subgroups $K_{n} \triangleleft G$ such that each quotient $G / K_{n}$ is a connected compact Lie group and $\bigcap_{n \geq 1} K_{n}=\{1\}$. Let $\mathcal{D}_{n}$ be the set of all pairs $(a, b)$ in $G \times G$ that generate a dense subgroup in the quotient $G / K_{n}$. Clearly $\mathcal{D}\left(F_{2}, G\right)=\bigcap \mathcal{D}_{n}$, and by Lemma 8.1, $\mathcal{D}_{n}$ is of second Baire category and of full Haar measure.

The analogous assertion for $\mathcal{F}\left(F_{2}, G\right)$ follows easily from Theorem 5.1 because one of the quotients $G / K_{n}$ is nonabelian.

Corollary 8.3 Any connected second countable nonabelian compact group contains a dense surface group of genus 2.

\section{References}

[1] M Abert, Y Glasner, Generic groups acting on a regular tree, preprint

[2] G Baumslag, On generalised free products, Math. Z. 78 (1962) 423-438 MR0140562

[3] E Breuillard, T Gelander, A topological Tits alternative, to appear in Annals of Math.

[4] E Breuillard, T Gelander, On dense free subgroups of Lie groups, J. Algebra 261 (2003) 448-467 MR1966638

[5] Y de Cornulier, A Mann, Some residually finite groups satisfying laws, to appear in the proceedings of the conference "Asymptotic and Probabilistic Methods in Geometric Group Theory”, Geneva (2005) 
[6] D B A Epstein, Almost all subgroups of a Lie group are free, J. Algebra 19 (1971) 261-262 MR0281776

[7] T Gelander, Y Glasner, Countable primitive groups, to appear in Geom. Funct. Anal.

[8] T Gelander, A Zuk, Dependence of Kazhdan constants on generating subsets, Israel J. Math. 129 (2002) 93-98 MR1910934

[9] V Kaloshin, The existential Hilbert 16-th problem and an estimate for cyclicity of elementary polycycles, Invent. Math. 151 (2003) 451-512 MR1961336

[10] A Lubotzky, B Weiss, Groups and expanders, from: "Expanding graphs (Princeton, NJ, 1992)”, DIMACS Ser. Discrete Math. Theoret. Comput. Sci. 10, Amer. Math. Soc., Providence, RI (1993) 95-109 MR1235570

[11] G A Margulis, Some remarks on invariant means, Monatsh. Math. 90 (1980) 233-235 MR596890

[12] D Montgomery, L Zippin, Topological transformation groups, Interscience Publishers, New York-London (1955) MR0073104

[13] Z Sela, Diophantine geometry over groups. I. Makanin-Razborov diagrams, Publ. Math. Inst. Hautes Études Sci. (2001) 31-105 MR1863735

[14] D Sullivan, For $n>3$ there is only one finitely additive rotationally invariant measure on the $n$-sphere defined on all Lebesgue measurable subsets, Bull. Amer. Math. Soc. (N.S.) 4 (1981) 121-123 MR590825

[15] J Tits, Free subgroups in linear groups, J. Algebra 20 (1972) 250-270 MR0286898

Université de Lille, UFR de Mathematiques, 59655 Villeneuve d'Ascq, FRANCE

Mathematics Department, Yale University, 10 Hillhouse ave, New Haven CT 06511, USA

Dept of Maths, University of Chicago, 5734 S. University Avenue, Chicago, IL 60637, USA

Stanford University, Mathematics, Bldg. 380, 450 Serra Mall, Stanford, CA 94305, USA

emmanuel.breuillard@math.univ-lille1.fr, tsachik.gelander@yale.edu, juan@math.uchicago.edu, storm@math.stanford.edu

Proposed: Benson Farb

Seconded: Jean-Pierre Otal, Walter Neumann
Received: 10 February 2006

Revised: 3 August 2006 